\title{
Interactive comment on "The use of GNSS zenith total delays in operational AROME/Hungary 3D-Var over a Central European domain" by Máté Mile et
} al.

\section{Anonymous Referee \#1}

Received and published: 14 January 2019

This paper presents results on the impact in terms of forecast scores of assimilating GNSS ZTD in a convective scale numerical weather prediction model within an experimental framework compatible with operational applications. Many papers have already been published on this subject during the last 15 years. An original aspect of the present study is the application over Central Europe with a specific network of surface stations. This study also examine the interest of using a variational bias correction scheme on the observations which has only been recently assessed by Sanchez-Ariola et al. (2016) and Lindskog et al. (2017). The presentation is clear and the forecast impacts on low level parameters and surface precipitation are consistent with previous published studies. My recommendation is to accept the publication once minor 
modifications suggested below are taken into account.

P1L12-13: replace "At the end of this article, the conclusion is drawn" by "Finally, AMTD conclusion are drawn"

P1L16: replace "System" by "Systems"

P2L2: replace "rely on more the remote" by " rely significantly on remote"

Interactive

comment

P2L4: replace "taken up" by "included"

P2L15: replace "employing" by "using"

P2L17-18: replace "Recently new European" by "Recently a new European"

P2L21: replace "All of these" by "All these"

P2L23: replace "error" by "errors"

P2L24: replace "assay of ZTD bias were examined" by "assessment of ZTD were proposed"

P2L25: replace "demonstrated the" by "demonstrated that the"

P2L29: replace "utilized" by " used"

P2L34: replace "the conclusion is drawn" by "conclusions are drawn"

P3L1: replace "Applied" by "Description of"

P3L3: replace "consortia" by "consortium"

P3L6: replace "Meso-NH(?)" by "Meso-NH (Lac et al., 2018)"

Lac, C., Chaboureau, J.-P., Masson, V., Pinty, J.-P., Tulet, P., Escobar, J., Leriche, M., Barthe, C., Aouizerats, B., Augros, C., Aumond, P., Auguste, F., Bechtold, P., Berthet, S., Bielli, S., Bosseur, F., Caumont, O., Cohard, J.-M., Colin, J., Couvreux, F., Cuxart, J., Delautier, G., Dauhut, T., Ducrocq, V., Filippi, J.-B., Gazen, D., Geoffroy, O., Gheusi,

Printer-friendly version

Discussion paper 
F., Honnert, R., Lafore, J.-P., Lebeaupin Brossier, C., Libois, Q., Lunet, T., Mari, C., Maric, T., Mascart, P., Mogé, M., Molinié, G., Nuissier, O., Pantillon, F., Peyrillé, P., AMTD Pergaud, J., Perraud, E., Pianezze, J., Redelsperger, J.-L., Ricard, D., Richard, E., Riette, S., Rodier, Q., Schoetter, R., Seyfried, L., Stein, J., Suhre, K., Taufour, M., Thouron, O., Turner, S., Verrelle, A., Vié, B., Visentin, F., Vionnet, V., and Wautelet, P.: Overview of the Meso-NH model version 5.4 and its applications, Geosci. Model Dev., 11, 1929-1969, https://doi.org/10.5194/gmd-11-1929-2018, 2018.

P3L10: replace "Later major upgrades consisting direct" by "Later, major upgrades consisting of a direct"

P3L12: replace "improvement" by "improvements"

P3L15: replace "(Mahfouf, 1991)" by "(Mahfouf, 1991; Masson et al. 2013)"

Masson, V., Le Moigne, P., Martin, E., Faroux, S., Alias, A., Alkama, R., Belamari, S., Barbu, A., Boone, A., Bouyssel, F., Brousseau, P., Brun, E., Calvet, J.-C., Carrer, D., Decharme, B., Delire, C., Donier, S., Essaouini, K., Gibelin, A.-L., Giordani, H., Habets, F., Jidane, M., Kerdraon, G., Kourzeneva, E., Lafaysse, M., Lafont, S., Lebeaupin Brossier, C., Lemonsu, A., Mahfouf, J.-F., Marguinaud, P., Mokhtari, M., Morin, S., Pigeon, G., Salgado, R., Seity, Y., Taillefer, F., Tanguy, G., Tulet, P., Vincendon, B., Vionnet, V., and Voldoire, A.: The SURFEXv7.2 land and ocean surface platform for coupled or offline simulation of earth surface variables and fluxes, Geosci. Model Dev., 6, 929-960, https://doi.org/10.5194/gmd-6-929-2013, 2013.

P3L15-16: remove "called OI_main"

P3L17: I suggest to provide the location of the ground based GNSS receivers on this map, instead of the model orography (that is not commented and not necessary)

P3L18: replace "employs" by "considers"

P4L1 : replace "appiled" by "applied" 
P4L4 : It is not clear what are the instruments that are used from NOAA-19 (AMSU-A only or AMSU-A and MHS ?)

P4L9: replace "(Girard, 1987)" by "(Girard, 1987; Chapnik et al., 2006)"

Chapnik, B., G. Desroziers, F. Rabier and O. Talagrand, 2006 : Diagnosis and tuning of observational error in a quasi-operational data assimilation setting. Quart. Jour. Roy. Meteor. Soc., $\mathrm{n}^{\circ}$ 615, Part B, volume 132, pp 543-565.

P4L11-12: replace the sentence by "where HK is the product of the linearized observation operator by the Kalman gain, and the DFS scores can be approximated by its trace as (1). The $y$ and y' are the unperturbed and perturbed observations sets"

P4L13: replace "states at the observation space" by "states in the observation space"

P4L19: replace "dates back" by "started in"

P4L20: replace "experiences" by "results"

P4L27: replace "provides the most of ZTD estimated" by "provides most of the ZTD estimates"

P4L30: remove "estimation for"

P5L1-2: Explain what is a relative DFS (division by the number of observations). A 2-D pie chart would be more readable

P5L3-4: As mentioned above (comment about Figure 1). A map showing the GNSS stations would be very useful

P5L7: replace "with increased observation error" by "with very large observation errors"

P5L8: put "passive" with quotes

P5L9: replace "without its influence on the analysis" by "without influencing the analysis"

Interactive

comment

Printer-friendly version

Discussion paper 
P5L10-13: I propose to reformulate this sentence that is not clear enough: "background check(which is dedicated to reject observations far from model background state) one AMTD also needs to ensure that only observations with Gaussian, zero mean and uncorrelated errors are selected in the assimilation (i.e. reliable stations). For that purpose, a specific pre-selection procedure has to be performed by checking passive observation minus first-guess (OMF) departures on a training period. Due to the increased"

Interactive comment

P5L13: "Due to the increased" : one can wonder with respect to what ? It would be better just to state "Due to the high"

P5L15: remove "examined"

P6L1-2: There is a need to clarify what you mean by "station multiplication". My understanding is that one specific station can be processed by several analysis centre and you have to select the best duple (station/analysis centre)

P6L2: replace "is also a part" by "is also part"

P6L3: replace "are written in" by "are given in"

P6L6-7: I propose to rewrite as follows: "The actual training period led to the availability of 197 GNSS stations inside the NWP domain (from three different networks). The preselection procedure excluded more than $30 \%$ of them, resulting in 122 trusted GNSS stations for active assimilation experiments"

P6L6-7: Again a map with the location of the stations (all + selected ones) would be useful

P6L8: replace "coverage issues" by "coverage"

P6L9: replace "than the pre-defined limits" by "than pre-defined limits"

P6L11: replace "station multiplication" by "multiple (station/analysis centre)" See similar comment above 
P6L12: "observation error correlations" by "horizontal observation error correlations"

P6L17: replace "having" by "considering"

P6L19: replace "cheap approach" by " efficient approach"

P6L19: replace "another whitelist is originally" by "a revised whitelist is" You should explain above what you mean by "whitelist" since it has not been defined before.

Interactive

comment

P6L22: replace "on Figure $320 \mathrm{~km}$ " by "in Figure 3, a $20 \mathrm{~km}$ " Why not using a curve fitting of the correlation function for an objective estimation of the thinning distance ?

P7L1 : The figure should be improved (horizontal axis unreadable) and the legend should be completed (provide information on the period and on the domain)

P7L5: replace "it presumes the" by "it assumes that the"

P7L7: replace "are plotted on Figure 4" by "is plotted in Figure 4"

P7L8: replace "on Figure 5 and 6 that observed" by "in Figure 5 and 6 that the observed"

P7L9: replace "Therefore bias correction" by "Therefore the bias correction"

P7L14: replace "experiments in the Section 5" by "experiments presented in Section 5"

P8L1: Enhance the legend (specify that it is ZTD, provide the period and the domain)

P8L2: Enhance the legend (specify that it is ZTD). The information given on the horizontal axis is totally unreadable.

P8L5: Provide a reference defining the stiffness parameter in terms of VARBC formulation (e.g. a paper by Dick Dee or the ECMWF documentation on data assimilation)

P8L7: replace "information in every new analysis" by "information at every analysis"

P9L1: The horizontal axis of the Figure is not readable. Specify that the quantity is 
ZTD. replace "purpule" by "purple"

P9L2: replace "prepare warmstart" by "prepare a warm start"

P9L9: replace "operational setup" by "operational setup (without ZTD observations". It is not clear to me if satellite observations are included in this "operational setup". The confusion comes from P3L18-19 defining the operational configuration. Have the satellite observations only been introduced for the DFS or also for this reference experiment ?

P9L10: replace "of operational observation set an static bias correction is compared with the reference" by "of the operational observation set an a static bias correction is compared to the reference".

P9L11: replace "employs variational" by "employs a variational"

P9L14: replace "traning" by "training"

P9L15: replace "till" by "until"

P9L18: replace "expected to seen in" by "expected to reflect more on"

P10L1: replace "Outline" by "Summary"

P10L1: specify in the text is EEGPS2 includes or not satellite data

P10L2: replace "on Figure 8" by "in Figure 8"

P10L5: replace "error reduction" by "error reduction with respect to the reference"

P10L5-6: "Furthermore, similar results are obtained with the static bias correction, but they are not statistically significant (not shown)"

P10L7: replace "on Figure 9" by "in Figure 9"

P10L7: replace "where the Equitable Threat Score (ETS) and the Symmetric" by "in terms of Equitable Threat Score (ETS) and Symmetric". Please provide a suitable

Interactive comment

Printer-friendly version

Discussion paper 
reference for the definition of the SEDI.

P10L8: replace " +12 hours precipitation forecasts can be seen" by "+12 hour forecast AMTD range"

P10L10: replace "has clear added value" by "has a clear added value". Given the smaller size of the sample for large precipitation amounts this result is maybe not sig-

Interactive nificant. Could you perform a statistical test of significance ?

comment

P10L14: The information on the use of 30 stations has to be put in the legend of Figure 7.

P10L14: replace "This is due to taking into" by "Taking into"

P10L15: replace "smaller impact of the relatively" by " a smaller impact given the relatively"

P10L15: replace "employed" by "assimilated"

P10L16: replace "the cause" by "another reason"

P10L16-17: The sentence needs to be clarified since the writing is awkward.

P10L19: replace "chosen to run the second" by "selected to perform a second"

P10L20: replace "as the summer trial" by "as for the summer trial"

P10L21: replace "applied for the" by "applied to the"

P10L22: replace "but has the possibility to" by "but allows to"

P11L1-2: indicate the statistics are performed using 30 SYNOP stations.

P11L4: replace "to static bias correction. Examining" by "to the static bias correction. By examining"

P11L4: replace "on Figure 10" by "in Figure 10"

Printer-friendly version

Discussion paper 
P11L5-6: replace "obtained in summer period is slightly remained for short-ranges" by "obtained for the summer period slightly remains at short-ranges"

P11L6: replace "slightly" by "slight"

P11L7: what do you mean by "for one or two forecast ranges" ?

Interactive

P11L8: what do you mean by "overfitting issues" ?

comment

P11L9: replace "were overestimated by the" by "are overestimated using the". If observation errors are too large, it should not lead to an "overfitting". Please clarify

P11L9: replace "the impact of other forecast parameters was" by "the impact on other forecast parameters is"

P11L10: replace "to summer" by "to the summer"

P11L11: replace "include" bu "show". replace "This is positive" by "The impact is positive"

P11L12: replace "3mm" by "3 $\mathrm{mm} "$

P11L12-13: "Similarly to the summer OSE, the value of an adaptive bias correction is confirmed by the results of the winter trial as well"

P11L14: replace "SYNOP stations, therefore overall results show neutral" by "SYNOP stations. Indeed, overall results show a neutral"

P12L5-6: reformulation of the sentence: "The potential and importance of this observation type was shown through DFS diagnostics. This is particularly relevant in data assimilation systems with a high frequency analysis cycle"

P12L7: replace "having potential" by "have the potential"

Printer-friendly version

P12L8: remove "As ZTD observations are by default blacklisted"

P12L8: replace "the pre-selection procedure of the trusted GNSS" by "A pre-selection 
P12L8-9: replace "has to be done" by "has been done"

P12L9: replace "sufficiently cover the wider area" by "cover sufficiently a wide area" (this has to be presented in a Figure plotting the stations)

Interactive

P12L12: replace "without risking" by "and to avoid"

comment

P12L13: what do you mean by "separate correction" ? a specific correction for each (station/analysis centre) ?

P12L14: replace "debiasing" by "removing the bias of"

P12L15: replace "GNSS data has positive" by "GNSS data have a positive"

P12L16: please clarify what you mean by "if bias information is also up-to-date" ? This was not the case in winter

P12L16: replace "The observation impact in the verification of" by "This positive impact on forecast scores during the"

P12L17: replace "using only" by "given the"

P13L1: replace "12 hours" by "12 hour". Replace "cummulation" by "accumulation"

P13L4: replace "when" by "using the". Remove "was employed"

P13L4: replace "but with" by "whereas with the"

P13L8: replace "concluded that" by "concluded that the"

P13L11: replace "smaller" by "small"

P13L13: "an improved stiffness parameter might be investigated in order to allow more flexibility to the system"

P16L1: replace "12 hours" by "12 hour". Replace "cummulation" by "accumulation" 
Interactive comment on Atmos. Meas. Tech. Discuss., doi:10.5194/amt-2018-433, 2018.

Interactive comment 